\title{
Heuristic Algorithms for Solving Multiobjective Transportation Problems
}

\author{
Ali Musaddak Delphi \\ Department of Mathematics, College of Basic education, University of Misan, Iraq. E-mail: alimathdelphi@yahoo.com
}

Received: April 4, 2016 Accepted: April 20, 2016 Online Published: May 9, 2016

doi:10.5539/jmr.v8n3p1 URL: http://dx.doi.org/10.5539/jmr.v8n3p1

\begin{abstract}
In this paper, we proposed three heuristic algorithms to solve multiobjective transportation problems, the first heuristic algorithm used to minimize two objective functions (total flow time and total late work), the second one used to minimize two objective functions (total flow time and total tardiness) and the last one used to minimize three objective functions (total flow time, total late work and total tardiness), where these heuristic algorithms which is different from to another existing algorithms and providing the support to decision markers for handing time oriented problems.
\end{abstract}

Keywords: Transportation problems, Heuristic algorithms, Feasible solutions, Multiobjective problems.

\section{Introduction}

Decision making is the process of identifying and choosing alternatives based on the values and preferences of the decision maker. It is the process of sufficiently reducing uncertainly and doubt about alternatives to allow a reasonable choice to be made from among them. Decision making based solely on a single criterion appears insufficient as soon as the decision making-making process deals with the complex organization environment. So, one must acknowledge the presence of several criteria that lead to the development of multicriteria decision making. Optimization is a kind of the decision making, in which decision have to be taken to optimize one or more objectives under some prescribes set of circumstances. These problems may be single or multiobjective and are to be optimized (maximized or minimized) under a specified set of constraints. The constraints usually are in the form of inequalities or equalities. Such problems which often arise as a result of mathematical modeling of many real life situations are called optimization problems. Transportation problem is a special type of optimization problems which arise in many practical applications. In the beginning it was founded for determining the optimal shipping patter, so it is called transportation problems. The conventional and very well known transportation problem consists in transporting a certain product from each of $\mathrm{m}$ origins $i=1, \ldots, m$ to any of $n$ destination $j=1, \ldots, n$. The origins are production facilities with respect capacities $a_{1}, \ldots, a_{m}$ and the destination are warehouse with required levels of demand $b_{1}, \ldots, b_{n}$. For the transport of a unit of the given product from the $i^{\text {th }}$ source to the $i^{\text {th }}$ destination a cost $C_{i j}$ is given for which, without loss of generally, we can assume $c_{i j} \geq 0$ for each $i$ and $j$. Hence, one must determine the amount $x_{i j}$ to be transported from all the origins $i=1, \ldots, m$ to all destination $j=1, \ldots, n$ in such a way that the total cost is minimized. This problem can be suitably modeled as a linear programming problem. Thus the conventional transportation problem can be mathematically expressed as:

Minimize $\quad z=\sum_{i=1}^{m} \sum_{j=1}^{n} C_{i j} X_{i j}$

Subject to $\quad \sum_{i=1}^{m} x_{i j}=b_{j}$ for all $j=1,2, \ldots, n$

$$
\begin{gathered}
\sum_{j=1}^{n} x_{i j}=a_{j} \quad \text { for all } \quad i=1,2, \ldots, m \\
x_{i j} \geq 0 \quad \text { for all } i \text { and } j \\
\sum_{i=1}^{m} a_{i}=\sum_{j=1}^{n} b_{j} \text { (Balanced condition) }
\end{gathered}
$$

In real world cases transportation problem can be formulated as a multiobjective transportation problem because the complexity of the social and economic environment requires the explicit consideration of criteria other than the cost. Examples of additional concerns include: average delivery time of the commodities, reliability of transportation, 
accessibility to the users, product deterioration, among others. Until now, many researchers also have great interest in the multiobjective transportation problem, and a number of methods had been proposed for solving it like (Ammar \& Youness, 2005) studied the multiobjective transportation problem, in which the cost factors, the sizes of supply and demand are all fuzzy numbers, (chakrobarty \& chakrobarty, 2010) presenting method for minimizing the cost and time for transportation problem for this they using linear membership function, (Mahapatra and et al., 2010) presented multiobjective stochastic unbalanced transportation problem, they used fuzzy programming techniques and stochastic method for randomness of sources and destination parameters in inequality type constraints, (Huseen and et al., 2011) introduced the possibilistic multiobjective transportation problem is considered possibilistic parameters with known distribution into multiobjective programming frame work, recently (Sharma and et al., 2015) have used new proposed algorithm to minimize cost and time of goods which is supply from one source to another source. It is clear from the literature review of algorithms that a limited amount of work has been done in the area of multiobjective transportation problems. The majority of the research work has been focused in the area of transportation two objective problem. In this paper we will provide solution to multiobjective transportation problems using new heuristic algorithms.

\section{Multiobjective Transportation Problem (MOTP)}

Standard MOTP is to transfer goods from several origins to different destination subject to linear constraints such that all objective optimal. Suppose there are (m) origins of goods denoted by $O_{1}, O_{2}, \ldots, O_{m}$ having supplies $a_{1}, a_{2}, \ldots, a_{m}$ and (n) destinations denoted by $D_{1}, D_{2}, \ldots, D_{n}$ having demands $b_{1}, b_{2}, \ldots, b_{m}$. We assume total demand is equal to total supply. Mathematically $\sum_{i=1}^{m} a_{i}=\sum_{j=1}^{n} b_{j}$. For each of the objective, $c_{i j}^{k}$ be the cost or penalty of transferring one unit from $i^{\text {th }}$ origin to $j^{\text {th }}$ destination such that $z_{k}$ is minimum for $k=1,2, \ldots, K$. A available $x_{i j}$ represent the unknown quantity to be shipped from $i^{\text {th }}$ source to $j^{\text {th }}$ destination. Thus MOTP is as under :

Minimize $z_{k}=\sum_{i=1}^{m} \sum_{j=1}^{n} C_{i j}^{k} X_{i j}$ for all $k=1,2, \ldots, K$

Subject to $\sum_{i=1}^{m} x_{i j}=b_{j}$ for all $j=1,2, \ldots, n$

$$
\begin{aligned}
& \sum_{j=1}^{n} x_{i j}=a_{j} \text { for all } i=1,2, \ldots, m \\
& x_{i j} \geq 0 \quad \text { for all } i \text { and } j
\end{aligned}
$$

In the sense of multiobjective transportation problems, objective functions are usually conflicting each other in nature and the concepts of optimal solution gives place to the concept of Pareto optimal (efficient, nondominated, noninferior) solutions, for which the improvement of one objective function is attained only by sacrificing another objective function. Denoted the feasible set in decision space by $F$, for the multicriteria optimization we can get the following definitions for Pareto optimal solution and ideal point.

Definition (1)(Huseen and et al., 2011) : A feasible solution $x^{*}=\left[x_{i j}^{*}\right]$ is said to be a Pareto optimal solution if and only if there does not exist another $x \in F$ such that $z_{k}(x) \leq z_{k}\left(x^{*}\right)$ for every $k=1,2, \ldots, K$ and $z_{p}(x) \neq z_{p}\left(x^{*}\right)$ for some $p$. If $x^{*}$ is efficient solution, then $z\left(x^{*}\right)$ is called a non-dominated point. The set of all efficient solutions $x \in F$ is called efficient set and the set of all non-dominated points $z\left(x^{*}\right)$ is called non-dominated set or Pareto front.

Definition(2)(Huseen and et al.,2011): the ideal point is one that optimize each objective function simultaneously. It can then be defined as $z^{*}=\left[z_{1}^{*}, z_{2}^{*}, \ldots, z_{k}^{*}\right]$ where $z_{k}^{*}$ is the optimal value for the $k-t h$ objective without considering other objectives.

\section{Heuristic Proposed Algorithms}

There are many algorithms that can be used for solving multiobjective transportation problems, which is to find the 
feasible solutions or at least approximation to it. The running time for the algorithm often increasing with the increase of the instance size. The purpose of any algorithm process is to find, for each instance a feasible solution called optimal, that minimize the objective function. This usual meaning of the optimum makes no sense in the multiobjective case because it doesn't exist, in most of the cases, a solution optimizing all objectives simultaneously. Hence we search for feasible solutions yielding the best compromise among objectives that constitutes a so called feasible solution set. These feasible solutions that cannot be improved in one objective without decreasing their performance in at least one of the others. It is clear that this feasible solutions set is difficult to find. Therefore, it could be preferable to have an approximation to that set in a reasonable amount of time. In this section, we shall try to find feasible solution for minimizing three objective transportation problem, where the first objective function is to minimize total flow time $F_{i j}$, the second objective function is to minimize total late work $V_{i j}$, where the late work for a task is the amount of processing of this task that is performed after its due date. and the last objective function is to minimize total tardiness $T_{i j}$, theses objective functions can be formulate into three transportation problems, the first transportation problem minimize two objective function total flow time $F_{i j}$ and total late work $V_{i j}$, and denoted by $\left(P_{1}\right)$. A mathematical model of MOTP with (2) objectives, (m) sources and (n) destinations can be written as: mathematically, the problem can be stated as

$$
\left.\begin{array}{ccc}
\text { Minimize } z_{1}= & \sum_{i=1}^{m} \sum_{j=1}^{n} x_{i j} F_{i j} \\
\text { Subject to } & z_{2}=\sum_{i=1}^{m} \sum_{j=1}^{n} x_{i j} V_{i j} x_{i j}=b_{j} \text { for all } j=1,2, \ldots, n \\
& \sum_{j=1}^{n} x_{i j}=a_{j} \quad \text { for all } i=1,2, \ldots, m \\
& x_{i j} \geq 0 \quad \text { for all } i \text { and } j
\end{array}\right\}
$$

The following heuristic algorithm (SCSVT) gives the feasible solution for problem $\left(P_{1}\right)$.

\section{Algorithm (1): Heuristic algorithm(SCTSE)}

Step (0): check the given problem is balanced

Step (1): we check the number of rows and columns are equal or not. If number of row are not equal to number of columns and vice versa. The dummy row or dummy column must be added with zero flow time/tardiness/late work elements with zero demand/supply, so our matrix becomes square matrix.

Step(2): put $V_{\mathrm{o}}=\left(\frac{\sum V_{i j}}{N}\right)-1$, where $N$ the number of the distinct $V_{i j}$ cell in the matrix.

Step(3): find cell $(I J)^{*} \in N$, such that $V_{(I J)^{*}} \leq V_{\circ}$, note that for this $V_{\circ}, \quad x_{i j}=O$ if $V_{i j} \cdot>V_{\circ}$ for each $i j \in N$ and solve the problem with minimum total completion time objective function $\left(F_{i j}\right)$, if $F_{(I J)^{*}}=F_{i J}$ choose the cell with smallest $V_{(I J)^{*}}$ if no cells with $V_{(I J)^{*}} \leq V_{\text {。 }}$ go to step(4).

Step (4): stop. 
Example(1): Consider the problem $\left(P_{1}\right)$ with the following data:

\begin{tabular}{|c|c|c|c|c|c|c|}
\hline \multicolumn{6}{|c|}{ DESTINATIONS } & \multirow{2}{*}{$\begin{array}{c}\text { SUPPLIES, } \\
\mathbf{A i}\end{array}$} \\
\hline & $\mathbf{i} / \mathbf{j}$ & B1 & B2 & B3 & B4 & \\
\hline \multirow{9}{*}{ SOURCES } & A1 & 4 & 10 & 11 & 35 & 25 \\
\hline & & 6 & 1 & 9 & 23 & \\
\hline & A2 & 38 & 25 & 10 & 49 & 15 \\
\hline & & 5 & 20 & 6 & 11 & \\
\hline & A3 & 19 & 8 & 25 & 23 & 15 \\
\hline & & 17 & 2 & 20 & 10 & \\
\hline & A4 & 11 & 12 & 15 & 13 & 30 \\
\hline & & 4 & 8 & 3 & 7 & \\
\hline & $\begin{array}{c}\text { DEMANDS, } \\
\text { bi }\end{array}$ & 20 & 25 & 25 & 15 & 85 \\
\hline
\end{tabular}

First, we calculate: $V_{\circ}=\left(\frac{\sum V_{i j}}{N}\right)-1 \Rightarrow V_{\circ}=\left(\frac{1+2+3+4+5+6+7+8+9+10+11+17+20+23}{14}\right)-1$, and so $V_{\circ}=\left(\frac{126}{14}\right)-1 \Rightarrow V_{\circ}=9-1=8$. Now, we ignore all cells that have $V_{i j} .>8$, and choose the remaining cells with lest completion time, as shown in the following table:

\begin{tabular}{|c|c|c|c|c|c|c|c|c|c|c|}
\hline & & \multicolumn{8}{|c|}{ DESTINATIONS } & \multirow{2}{*}{$\begin{array}{c}\text { SUPPLIES, } \\
\text { ai } \\
\end{array}$} \\
\hline & $\mathbf{i} / \mathbf{j}$ & & B1 & & B2 & & B3 & & B4 & \\
\hline \multirow{9}{*}{ SOURCES } & A1 & 15 & 4 & 10 & 10 & & 11 & & 35 & 25 \\
\hline & & & 6 & & 1 & & 9 & & 23 & \\
\hline & A2 & & 38 & & 25 & 10 & 10 & & 49 & 15 \\
\hline & & & 5 & & 20 & & 6 & & 11 & \\
\hline & A3 & & 19 & 15 & 8 & & 25 & & 23 & 15 \\
\hline & & & 17 & & 2 & & 20 & & 10 & \\
\hline & A4 & 5 & 11 & & 12 & 15 & 15 & 15 & 13 & 30 \\
\hline & & & 4 & & 8 & & 3 & & 7 & \\
\hline & $\begin{array}{c}\text { DEMANDS, } \\
\text { bi }\end{array}$ & & & & & & & & & 85 \\
\hline
\end{tabular}

then we have the following feasible solutions for each objective fuctions:

$$
\begin{aligned}
& z_{1}=\sum \sum x_{i j} F_{i j}=(15 \times 4)+(10 \times 10)+(10 \times 10)+(15 \times 8)+(5 \times 11)+(15 \times 15)+(15 \times 13)=775 \\
& z_{2}=\sum \sum x_{i j} V_{i j}=(15 \times 6)+(10 \times 1)+(10 \times 6)+(15 \times 2)+(5 \times 4)+(15 \times 3)+(15 \times 7)=360 .
\end{aligned}
$$

the second transportation problem also minimize two objective function total flow time $F_{i j}$ and total tardiness $T_{i j}$, and denoted by $\left(P_{2}\right)$. A mathematical model of MOTP with (2) objectives, (m) sources and (n) destinations can be written as: mathematically, the problem can be stated as

$$
\begin{aligned}
\text { Minimize } z_{1} & =\sum_{i=1}^{m} \sum_{j=1}^{n} x_{i j} F_{i j} \\
z_{2} & =\sum_{i=1}^{m} \sum_{j=1}^{n} x_{i j} T_{i j}
\end{aligned}
$$

Subject to

$$
\begin{array}{ccc}
\sum_{i=1}^{m} x_{i j}=b_{j} & \text { for all } & j=1,2, \ldots, n \\
\sum_{j=1}^{n} x_{i j}=a_{j} & \text { for all } & i=1,2, \ldots, m \\
x_{i j} \geq 0 & \text { for all } i \text { and } j
\end{array}
$$


The following heuristic algorithm (SCSTV) gives the feasible solution for $\operatorname{problem}\left(P_{2}\right)$.

Algorithm (2): Heuristic algorithm(SCSTV)

Step (0): check the given problem is balanced

Step (1): we check the number of rows and columns are equal or not. If number of row are not equal to number of columns and vice versa. The dummy row or dummy column must be added with zero flow time/tardiness/late work elements with zero demand/supply, so our matrix becomes square matrix.

Step(2): put $T_{\circ}=\left(\frac{\sum T_{i j}}{N}\right)-1$, where $N$ the number of the distinct $T_{i j}$ cell in the matrix.

Step(3): find cell $(I J)^{*} \in N$, such that $T_{(I J)^{*}} \leq T_{\circ}$, note that for this $T_{\circ}, x_{i j}=O$ if $T_{i j}$. $>T_{\circ}$ for each $i j \in N$ and solve the problem with minimum total completion time objective function $\left(F_{i j}\right)$, if $F_{(I J)^{*}}=F_{i J}$ choose the cell with smallest $T_{(I J)^{*}}$, if no cells with $T_{(I J)^{*}} \leq T_{\text {。 }}$ go to step(4).

Step (4):stop.

Example(2): Consider the problem $\left(P_{2}\right)$ with the following data:

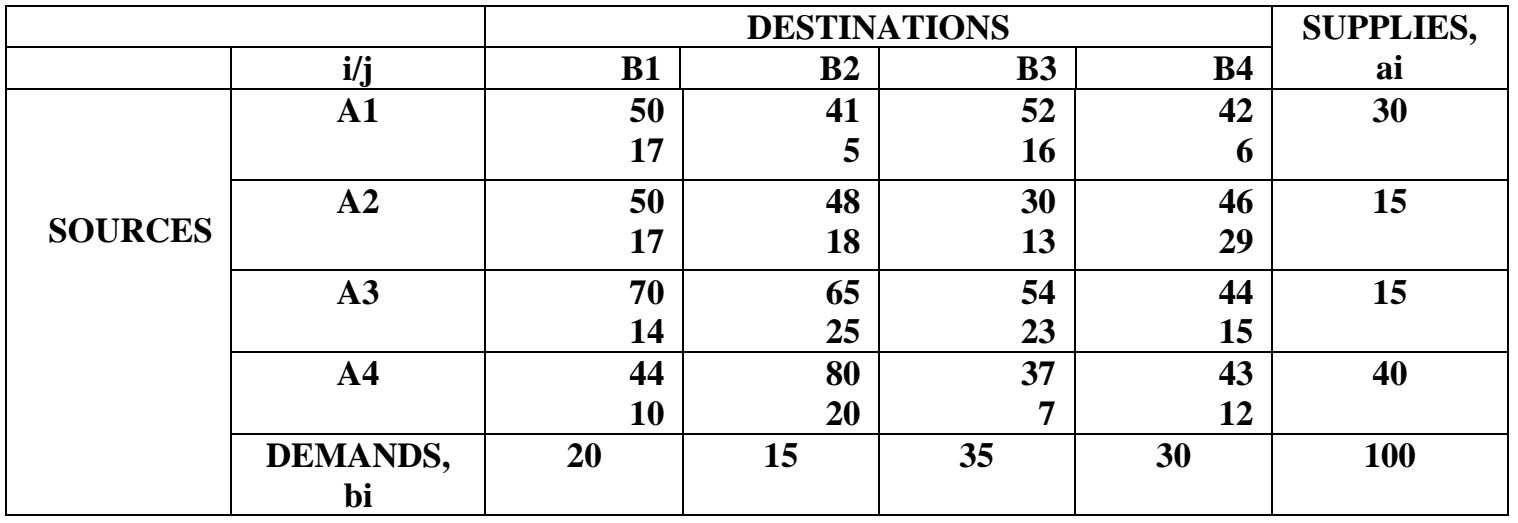

First we calculate: $T_{\circ}=\left(\frac{\sum T_{i j}}{N}\right)-1 \Rightarrow T_{\circ}=\left(\frac{5+6+7+10+12+13+14+15+16+17+18+20+23+25+29}{15}\right)-1$, and so $T_{\circ}=\left(\frac{230}{15}\right)-1 \Rightarrow T_{\circ}=15-1=14$. Now, we ignore all cells that have $T_{i j}>14$, and choose the remaining cells with lest completion time, as shown in the following table:

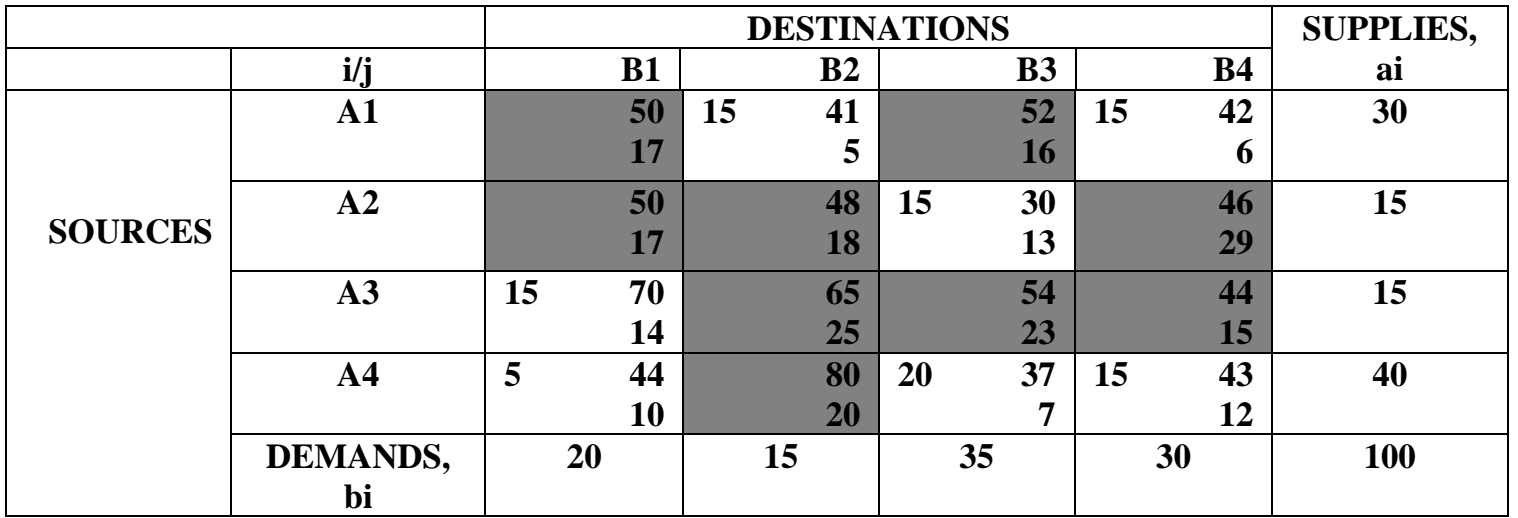

then we have the following feasible solutions for each objective functions: 
$z_{1}=\sum \sum x_{i j} F_{i j}=(15 \times 41)+(15 \times 42)+(15 \times 30)+(15 \times 70)+(5 \times 44)+(20 \times 37)+(15 \times 43)=4350$

$z_{2}=\sum \sum x_{i j} T_{i j}=(15 \times 5)+(15 \times 6)+(15 \times 13)+(15 \times 14)+(5 \times 10)+(20 \times 7)+(15 \times 12)=940$.

the last transportation problem minimize three objective function total flow time $F_{i j}$, total late work $V_{i j}$ and total tardiness $T_{i j}$, and denoted by $\left(P_{3}\right)$. A mathematical model of MOTP with (3) objectives, (m) sources and (n) destinations can be written as: mathematically, the problem can be stated as

Minimize

$$
\begin{aligned}
z_{1} & =\sum_{i=1}^{m} \sum_{j=1}^{n} x_{i j} F_{i j} \\
z_{2} & =\sum_{i=1}^{m} \sum_{j=1}^{n} x_{i j} V_{i j} \\
z_{3} & =\sum_{i=1}^{m} \sum_{j=1}^{n} x_{i j} T_{i j}
\end{aligned}
$$

Subject to

$$
\begin{aligned}
& \sum_{i=1}^{m} x_{i j}=b_{j} \quad \text { for all } \quad j=1,2, \ldots, n \\
& \sum_{j=1}^{n} x_{i j}=a_{j} \quad \text { for all } \quad i=1,2, \ldots, m \\
& x_{i j} \geq 0 \quad \text { for all } i \text { and } j
\end{aligned}
$$

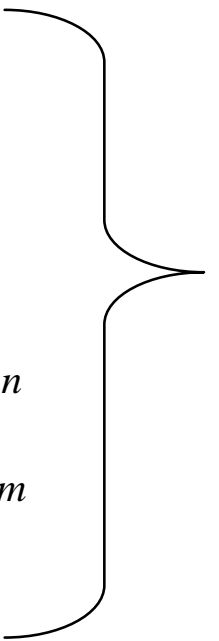

The following heuristic algorithm (SCSVST) gives the feasible solution for problem $\left(P_{3}\right)$.

Algorithm (3): Heuristic algorithm(SCSVST)

Step (0): check the given problem is balanced

Step (1): we check the number of rows and columns are equal or not. If number of row are not equal to number of columns and vice versa. The dummy row or dummy column must be added with zero flow time/tardiness/late work elements with zero demand/supply, so our matrix becomes square matrix.

Step(2): compute $V_{\circ}=\left(\frac{\sum V_{i j}}{N}\right)-1$ and $T_{\circ}=\left(\frac{\sum T_{i j}}{N}\right)-1$, where $N$ the number of the distinct $V_{i j}, T_{i j}$ cells in the matrix and put $\Omega=\min \left\{V_{\circ}, T_{\circ}\right\}$.

Step(3): if $\Omega=V_{\circ}$, find cell $(I J)^{*} \in N$, such that $T_{(I J)^{*}} \leq \Omega$, note that for this $\Omega, x_{i j}=O$ if $T_{i j}$. $>\Omega$ for each $i j \in N$ and solve the problem with minimum total completion time objective function $\left(F_{i j}\right)$, if $\Omega=T_{\circ}$, find cell $(I J)^{*} \in N$, such that $V_{(I J)^{*}} \leq \Omega$, note that for this $\Omega, \quad x_{i j}=o$ if $V_{i j} .>\Omega$ for each $i j \in N$ and solve the problem with minimum total completion time objective function $\left(F_{i j}\right)$, if $F_{(I J)^{*}}=F_{i J}$ choose the cell with smallest $T_{(I J)^{*}}$ and if $T_{(I J)^{*}}=T_{i j}$ choose the cell with smallest $V_{(I J)^{*}}$, if no cells with $T_{(I J)^{*}} \leq T_{\text {。 }}$ go to step(4).

Step (4):stop.

Example(3): Consider the problem $\left(P_{3}\right)$ with the following data: 


\begin{tabular}{|c|c|c|c|c|c|c|}
\hline & & \multicolumn{4}{|c|}{ DESTINATIONS } & \multirow{2}{*}{$\begin{array}{c}\text { SUPPLIES, } \\
\text { ai }\end{array}$} \\
\hline & $\mathbf{i} / \mathbf{j}$ & B1 & B2 & B3 & B4 & \\
\hline \multirow{13}{*}{ SOURCES } & \multirow[t]{3}{*}{ A1 } & 4 & 10 & 11 & 35 & \multirow[t]{3}{*}{20} \\
\hline & & 1 & 9 & 9 & 27 & \\
\hline & & 6 & 1 & 1 & 20 & \\
\hline & \multirow[t]{3}{*}{ A2 } & 38 & 25 & 10 & 49 & \multirow[t]{3}{*}{10} \\
\hline & & 32 & 22 & 6 & 42 & \\
\hline & & 13 & 15 & 6 & 11 & \\
\hline & \multirow[t]{3}{*}{ A3 } & 19 & 8 & 25 & 35 & \multirow[t]{3}{*}{15} \\
\hline & & 17 & 2 & 22 & 28 & \\
\hline & & 11 & 2 & 6 & 10 & \\
\hline & \multirow[t]{3}{*}{ A4 } & 10 & 12 & 15 & 13 & \multirow[t]{3}{*}{35} \\
\hline & & 6 & 5 & 6 & 7 & \\
\hline & & 6 & 2 & 3 & 2 & \\
\hline & $\begin{array}{c}\text { DEMANDS, } \\
\text { bi }\end{array}$ & 25 & 25 & 15 & 15 & 80 \\
\hline
\end{tabular}

First we calculate:

$T_{\circ}=\left(\frac{\sum T_{i j}}{N}\right)-1 \Rightarrow T_{\circ}=\left(\frac{1+2+5+6+7+9+17+22+27+28+32}{15}\right)-1, \quad$ and $\quad$ so

$T_{\circ}=\left(\frac{156}{11}\right)-1 \Rightarrow T_{\circ}=14-1=13 . V_{\circ}=\left(\frac{\sum V_{i j}}{N}\right)-1 \Rightarrow V_{\circ}=\left(\frac{1+2+3+6+10+11+13+15+20}{9}\right)-1$, and so $V_{\circ}=\left(\frac{81}{9}\right)-1 \Rightarrow V_{\circ}=9-1=8$. Now, $\Omega=\min \left\{V_{\circ}, T_{\circ}\right\}=\min \{8,13\}=8, \quad$ we ignore all cells that have $T_{i j} .>8$,

and choose the remaining cells with lest completion time, as shown in the following table:

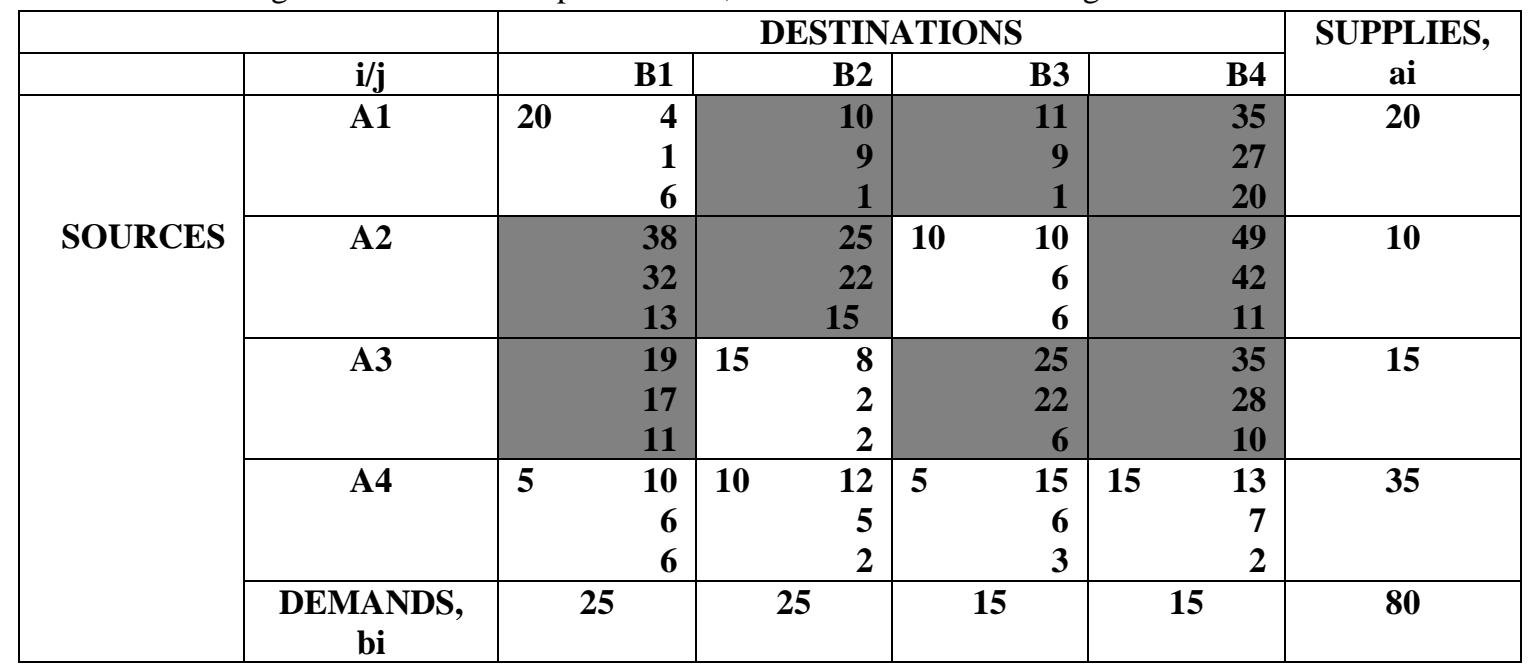

then we have the following feasible solutions for each objective functions:

$z_{1}=\sum \sum x_{i j} F_{i j}=(20 \times 4)+(10 \times 10)+(15 \times 8)+(5 \times 10)+(10 \times 12)+(5 \times 15)+(15 \times 13)=740$

$z_{2}=\sum \sum x_{i j} T_{i j}=(20 \times 4)+(10 \times 6)+(15 \times 2)+(5 \times 6)+(10 \times 5)+(5 \times 6)+(15 \times 7)=385$

$z_{2}=\sum \sum x_{i j} V_{i j}=(20 \times 6)+(10 \times 6)+(15 \times 2)+(5 \times 6)+(10 \times 2)+(5 \times 3)+(15 \times 2)=275$

\section{Conclusion}

In this paper, we conclude that the three heuristic algorithms found feasible solutions for the three problems optimality, these heuristic algorithms can be applied to different field such as military affair. It can be seen from the numerical examples that these heuristic algorithms provided in this paper is easier to compute and the results obtained are better. 


\section{References}

Ammar, E. E., \& Youness, E. A. (2005). Study on multi-objective transportation problem wih fuzzy numbers. Applies mathematics and computation, 166, 241-253. http://dx.doi.org/10.1016/j.amc.2004.04.103

Chakraborty, A., \& Chakraborty, M. (2010). Cost -time minimization in a transportation problem with fuzzy parameters: A case study. Journal of Transpn. Sys. Engg. And IT., 10(6), 53-63. http://dx.doi.org/10.1016/s1570-6672(09)60071-4

Huseen, M. L., Elbana, A. H., \& Gaafer, A. G . (2011). Possibilistic multi-objective transportation problem. International journal of optimization: theory, methods and applications, 3(1), 1-20.

Mahapatra, D. R., Roy, S. K., \& Biswal, M. P. (2010). Stochastic based multi-objective transportation problem involving normal randomness. Advance modeling and optimization journal, 12(2), 205-223.

Sharma, G., Abbas, S. H., \& Gupta, V. K. (2015). Solving multi-objective transportation problem to reduce transportation cost and time. Journal of advances in mathematics, 11(1), 3908-3912.

\section{Copyrights}

Copyright for this article is retained by the author(s), with first publication rights granted to the journal.

This is an open-access article distributed under the terms and conditions of the Creative Commons Attribution license (http://creativecommons.org/licenses/by/3.0/). 